EPJ Web of Conferences 85, 02009 (2015)

DOI: $10.1051 /$ epjconf/ 20158502009

(C) Owned by the authors, published by EDP Sciences, 2015

\title{
Quark Orbital Angular Momentum
}

\author{
Matthias Burkardt ${ }^{1, a}$ \\ ${ }^{1}$ Department of Physics, New Mexico State University, Las Cruces, NM 88003, U.S.A.
}

\begin{abstract}
Definitions of orbital angular momentum based on Wigner distributions are used as a framework to discuss the connection between the Ji definition of the quark orbital angular momentum and that of Jaffe and Manohar. We find that the difference between these two definitions can be interpreted as the change in the quark orbital angular momentum as it leaves the target in a DIS experiment. The mechanism responsible for that change is similar to the mechanism that causes transverse single-spin asymmetries in semi-inclusive deep-inelastic scattering.
\end{abstract}

\section{Introduction}

Since the famous EMC experiments revealed that only a small fraction of the nucleon spin is due to quark spins [1], there has been a great interest in 'solving the spin puzzle', i.e. in decomposing the nucleon spin into contributions from quark/gluon spin and orbital degrees of freedom. In this effort, the Ji decomposition [2]

$$
\frac{1}{2}=\frac{1}{2} \sum_{q} \Delta q+\sum_{q} L_{q}^{z}+J_{g}^{z}
$$

appears to be very useful: through GPDs, not only the quark spin contributions $\Delta q$ but also the quark total angular momenta $J_{q} \equiv \frac{1}{2} \Delta q+L_{q}^{z}$ (and by subtracting the spin piece also the the quark orbital angular momenta $L_{q}^{z}$ ) entering this decomposition can be accessed experimentally. The terms in (1) are defined as expectation values of the corresponding terms in the angular momentum tensor

$$
M^{0 x y}=\sum_{q} \frac{1}{2} q^{\dagger} \Sigma^{z} q+\sum_{q} q^{\dagger}\left(\vec{r} \times \frac{1}{i} \vec{D}\right)^{z} q+[\vec{r} \times(\vec{E} \times \vec{B})]^{z}
$$

in a nucleon state polarized in the $+\hat{z}$ direction. Here $\vec{D}=\vec{\partial}+i g \vec{A}$ is the gauge-covariant derivative. The main advantages of this decomposition are that each term can be expressed as the expectation value of a manifestly gauge invariant local operator and that the quark total angular momentum $J^{q}=\frac{1}{2} \Delta q+L^{q}$ can be related to GPDs [2] and is thus accessible in deeply virtual Compton scattering and deeply virtual meson production and can also be straightforwardly calculated in lattice gauge theory. Recent lattice calculations of GPDs [3] yielded the surprising result that the light quark orbital angular momentum (OAM) is consistent with $L^{u} \approx-L^{d}$, i.e. $L^{u}+L^{d} \approx 0$. Unless there is a large contribution from disconnected quark loops, that had been so far omitteed, this would imply that $J^{g} \approx \frac{1}{2} \cdot 0.7$

\footnotetext{
a e-mail: burkardt@nmsu.edu
}

represents the largest piece in the nucleon spin decomposition.

Jaffe and Manohar have proposed an alternative decomposition of the nucleon spin, which does have a partonic interpretation [4], and in which also two terms, $\frac{1}{2} \Delta q$ and $\Delta G$, are experimentally accessible

$$
\frac{1}{2}=\frac{1}{2} \sum_{q} \Delta q+\sum_{q} \mathcal{L}^{q}+\Delta G+\mathcal{L}^{g} .
$$

The individual terms in (3) can be defined as matrix elements of the corresponding terms in the +12 component of the angular momentum tensor

$$
\begin{aligned}
M^{+12} & =\frac{1}{2} \sum_{q} q_{+}^{\dagger} \gamma_{5} q_{+}+\sum_{q} q_{+}^{\dagger}\left(\vec{r} \times \frac{1}{i} \vec{\partial}\right)^{z} q_{+} \\
& +\varepsilon^{+-i j} \operatorname{Tr} F^{+i} A^{j}+2 \operatorname{Tr} F^{+j}\left(\vec{r} \times \frac{1}{i} \vec{\partial}\right)^{z} A^{j}
\end{aligned}
$$

for a nucleon polarized in the $+\hat{z}$ direction. The first and third term in $(3,4)$ are the 'intrinsic' contributions (no factor of $\vec{r} \times$ ) to the nucleon's angular momentum $J^{z}=+\frac{1}{2}$ and have a physical interpretation as quark and gluon spin respectively, while the second and fourth term can be identified with the quark/gluon OAM. Here $q_{+} \equiv \frac{1}{2} \gamma^{-} \gamma^{+} q$ is the dynamical component of the quark field operators, and light-cone gauge $A^{+} \equiv A^{0}+A^{z}=0$ is implied. The residual gauge invariance can be fixed by imposing anti-periodic boundary conditions $\overrightarrow{A_{\perp}}\left(\mathbf{x}_{\perp}, \infty\right)=-\overrightarrow{A_{\perp}}\left(\mathbf{x}_{\perp},-\infty\right)$ on the transverse components of the vector potential. $\mathcal{L} \equiv \mathcal{L}_{g}+$ $\sum_{q} \mathcal{L}_{q}$ also naturally arises in a light-cone wave function description of hadron states, where $\frac{1}{2}=\frac{1}{2} \sum_{q} \Delta q+\Delta G+\mathcal{L}$, in the sense of an eigenvalue equation, is manifestly satisfied for each Fock component individually [5]. In Ref. [6] it was shown explicitly in QED that $L_{q} \neq \mathcal{L}_{q}$. However, it has remained misterious what the physical interpretation of the difference between the two orbital angular momenta is. For a recent review see Ref. [7]. 
Other decompositions, in which only one term is experimentally accessible, will not be discussed in this brief note.

\section{TMDs and Orbital Angular Momentum from Wigner Distributions}

Wigner distributions can be defined as defined as off forward matrix elements of non-local correlation functions [8-10]

$$
\begin{aligned}
& W^{\mathcal{U}}\left(x, \vec{b}_{\perp}, \vec{k}_{\perp}\right) \equiv \int \frac{d^{2} \vec{q}_{\perp}}{(2 \pi)^{2}} \int \frac{d^{2} \xi_{\perp} d \xi^{-}}{(2 \pi)^{3}} e^{-i \vec{q}_{\perp} \cdot \vec{b}_{\perp}} e^{i\left(x P^{+} \xi^{-}-\vec{k}_{\perp} \cdot \vec{\xi}_{\perp}\right)} \\
&\left\langle P^{\prime} S^{\prime}\left|\bar{q}(0) \Gamma \mathcal{U}_{0 \xi} q(\xi)\right| P S\right\rangle
\end{aligned}
$$

with $P^{+}=P^{+\prime}, P_{\perp}=-P_{\perp}^{\prime}=\frac{q_{\perp}}{2}$. Throughout this paper, we will chose $\vec{S}=\vec{S}^{\prime}=\hat{\vec{z}}$. Furthermore, we will focus on the 'good' component by selecting $\Gamma=\gamma^{+}$. In order to ensure manifest gauge invariance, a Wilson line gauge link $\mathcal{U}_{0 \xi}$ connecting the quark field operators at position 0 and $\xi$ must be included. The issue of choice of path for the Wilson line will be addressed below.

In terms of Wigner distributions, quark transverse momentum and OAM can be defined respectively as [12]

$$
\begin{aligned}
\left\langle\vec{k}_{\perp}\right\rangle_{\mathcal{U}} & =\int d x d^{2} \vec{b}_{\perp} d^{2} \vec{k}_{\perp} \vec{k}_{\perp} W^{\mathcal{U}}\left(x, \vec{b}_{\perp}, \vec{k}_{\perp}\right) \\
L_{\mathcal{U}} & =\int d x d^{2} \vec{b}_{\perp} d^{2} \vec{k}_{\perp}\left(\vec{b}_{\perp} \times \vec{k}_{\perp}\right)^{z} W^{\mathcal{U}}\left(x, \vec{b}_{\perp}, \vec{k}_{\perp}\right) .
\end{aligned}
$$

No issues with the Heisenberg uncertainty principle arise here since only perpendicular combinations of position $\vec{b}_{\perp}$ and momentum $\vec{k}_{\perp}$ are needed simultaneously in order to evaluate the integral for $L_{\mathcal{U}}$.

A straight line connecting 0 and $\xi$ for the Wilson line in $\mathcal{U}_{0 \xi}$ is often the most natural choice, resulting in [10]

$$
\begin{aligned}
L_{\text {straight }}^{q} & \equiv \int d x d^{2} \vec{b}_{\perp} d^{2} \vec{k}_{\perp}\left(\vec{b}_{\perp} \times \vec{k}_{\perp}\right)^{z} W^{\text {straight }}\left(x, \vec{b}_{\perp}, \vec{k}_{\perp}\right) \\
& =\frac{\int d^{3} \vec{r}\left\langle P S\left|q^{\dagger}(\vec{r})\left(\vec{r} \times \frac{1}{i} \vec{D}\right) q(\vec{r})^{z}\right| P S\right\rangle}{\langle P S \mid P S\rangle}=L_{J i}^{q} .
\end{aligned}
$$

Likewise, Wigner distributions employing light-like staples (Fig. 1) yield (in $A^{+}=0$ gauge)

$$
\begin{aligned}
\mathcal{L}_{ \pm}^{q} & \equiv \int d x d^{2} \vec{b}_{\perp} d^{2} \vec{k}_{\perp}\left(\vec{b}_{\perp} \times \vec{k}_{\perp}\right)^{z} W^{ \pm L C}\left(x, \vec{b}_{\perp}, \vec{k}_{\perp}\right) \\
& =\frac{\int d^{3} \vec{r}\langle P S| \bar{q}(\vec{r}) \gamma^{+}\left[\vec{r} \times\left(\frac{1}{i} \vec{\partial}-g \vec{A}_{\perp}\left( \pm \infty, \vec{r}_{\perp}\right)\right]^{z} q(\vec{r})|P S\rangle\right.}{\langle P S \mid P S\rangle}
\end{aligned}
$$

The shape of the segment at $\infty$ is irrelevant as the gauge field is pure gauge there, but it is still necessary to include a connection at $\infty$ and for simplicity we pick a straight line [11].

\section{Jaffe-Manohar-Bashinsky}

Eq. (8) differs from

$$
\mathcal{L}^{q}=\frac{\int d^{3} \vec{r}\left\langle P S\left|\bar{q}(\vec{r}) \gamma^{+}\left(\vec{r} \times \frac{1}{i} \vec{\partial}\right)^{z} q(\vec{r})\right| P S\right\rangle}{\langle P S \mid P S\rangle}
$$

Figure 1. Illustration of the path for the Wilson line gauge link used to define the Wigner distribution with light-cone staples.

(denoted $\tilde{L}^{q}$ in Ref. [10]) by the contribution from the gauge field $\overrightarrow{A_{\perp}}$ at $\pm \infty$.

PT invariance implies that $\mathcal{L}_{+}=\mathcal{L}_{-}$[13]. If antiperiodic boundary conditions are imposed, i.e. $\vec{A}_{\perp}(-$ $\left.\infty, \vec{r}_{\perp}\right)=-\vec{A}_{\perp}\left(\infty, \vec{r}_{\perp}\right)$ PT invariance thus implies that the vector potential at $\pm \infty$ does not contribute to $\mathcal{L}_{ \pm}$-in contradistinction to the average transverse momentum.

The so-called light-cone gauge $A^{+}=0$ does not completely fix the gauge as $A^{+}=0$ is not affected by transformations that depend on $\vec{r}_{\perp}$ only and thus affect $\vec{A}_{\perp}$. As a result also the $\hat{z}$ component of the orbital angular momentum is affected by these 'residual' gauge transformations. A common remedy is to impose anti-periodic boundary conditions

$$
\overrightarrow{A_{\perp}}\left(-\infty, \vec{x}_{\perp}\right)=-\vec{A}_{\perp}\left(-\infty, \vec{x}_{\perp}\right),
$$

which fixes the gauge symmetry. ${ }^{1}$ Having thus completely fixed the gauge even matrix elements of operators that are not manifestly gauge invariant, such as $\mathcal{L}_{q}$ will thus be uniquely defined.

PT invariance implies that $\mathcal{L}_{+}=\mathcal{L}_{-}$[13]. If antiperiodic boundary conditions are imposed (10) PT invariance thus implies that the vector potential at $\pm \infty$ does not contribute to $\mathcal{L}_{ \pm}$-in contradistinction to the average transverse momentum. As a result $\mathcal{L}_{ \pm}$becomes identical to the the Jaffe-Manohar definition $\mathcal{L}$.

As an alternative to imposing boundary conditions on $\overrightarrow{A_{\perp}}$ it was proposed in Ref. [14] to consider instead transverse derivatives that are manifestly invariant under residual gauge transformations which leave $A^{+}=0$ invariant

$$
i \partial_{\perp} \longrightarrow i \partial_{\perp}-g \mathcal{A}_{\perp}\left(\vec{x}_{\perp}\right)
$$

where

$$
\begin{aligned}
\mathcal{A}_{\perp}\left(\vec{x}_{\perp}\right) & \equiv \lim _{L \rightarrow \infty} \frac{1}{2 L} \int_{-L}^{L} d x^{-} A_{\perp}\left(x^{-}, \vec{x}_{\perp}\right) \\
& =\frac{1}{2}\left[A_{\perp}\left(\infty, \vec{x}_{\perp}\right)+A_{\perp}\left(-\infty, \vec{x}_{\perp}\right)\right],
\end{aligned}
$$

where the limit exists provided $\lim _{x^{-} \rightarrow \pm \infty} G^{+\perp}\left(x^{-}, \vec{x}_{\perp}\right)=0$, i.e. $\lim _{x^{-} \rightarrow \pm \infty} A_{\perp}\left(x^{-}, \vec{x}_{\perp}\right)$ exists.

Therefore, the quark orbital angular momentum $\mathcal{L}_{B J}$ defined using the above prescription as the matrix element

\footnotetext{
${ }^{1}$ It is not possible to impose periodic boundary conditions in $A^{+}=$ 0 gauge as the corresponding gauge transformation is not possible if $\int_{-\infty}^{\infty} d x^{-} F^{+\perp}\left(x^{-}, \vec{x}_{\perp}\right) \neq 0$.
} 
of $\bar{q} \gamma^{+}[\vec{r} \times(i \vec{\partial}-\overrightarrow{g A})] q$ satisfies

$$
\mathcal{L}_{B J}=\frac{1}{2}\left[\mathcal{L}_{+}+\mathcal{L}_{-}\right]=\mathcal{L}_{+}=\mathcal{L}_{-}
$$

and agrees, for antiperiodic boundary conditions, with $\mathcal{L}$.

\section{OAM and Final State Interactions}

In light-cone gauge, one can write

$$
A^{i}\left(\infty, \vec{x}_{\perp}\right)=A^{i}\left(x^{-}, \vec{x}_{\perp}\right)+\int_{x^{-}}^{\infty} d r^{-} F^{+i}\left(r^{-}, \vec{x}_{\perp}\right),
$$

i.e. the difference between the Ji definition of quark OAM (local manifestly gauge covariant derivative) and the JaffeManohar definition (light-cone staple) can be expressed in terms of a correlation between the quark density and an integral involving the gluon field strength tensor

$$
\mathcal{L}^{q}-L^{q}=-g \int d^{3} x\left\langle P, S\left|\bar{q}(\vec{x}) \gamma^{+}\left[\vec{x} \times \int_{x^{-}}^{\infty} d r^{-} F^{+\perp}\left(r^{-}, \mathbf{x}_{\perp}\right)\right]^{z} q(\vec{x})\right| P, S\right\rangle .
$$

It truns out that in the abelian case Eq. (15) is valid in an arbitrary gauge provided $\mathcal{L}^{q}$ is defined using light-cone staples. In the non-abelian case additional gauge links appear [15].

The matrx element on the r.h.s. of Eq. (15) has a very simple physical interpretation:

$$
\sqrt{2} F^{+y}=F^{0 y}+F^{z y}=-E^{y}+B^{x}
$$

represents the $\hat{y}$-component of the (color) Lorentz force acting on a particle that moves with the velocity of light in the $-\hat{z}$ direction (the direction of the large momentum transfer in DIS!). Up to a cross product with the transverse position vector, Eq. (15) resembles the Qiu Sterman matrix element [16] which has a semi-classical interpretation as the transverse impulse from the final state interactions on the active quark [17] and which gives rise to transverse single-spin asymmertries [18].

Eq. (15) thus has the interpretation as the change in orbital angular momentum due to the torque from the final state interactions on the escaping quark. In other words, while Ji's OAM represents the $\hat{z}$ component of the OAM before it was struck by the virtual photon, the JaffeManohar OAM represents the $\hat{z}$ component of the OAM after the quark has escaped along the $-\hat{z}$ direction. This change in orbital angular momentum is also identical to the 'potential angular momentum' in Ref. [19].

These observations also provide a guide on how to calculate the Jaffe-Manohar orbital angular momentum of quarks in lattice QCD: In a pioneering work, Ref. [20] demonstrated that it is possible to include final state interactions in lattice calculations for TMDs by studying matrix elements involving space-like staple-shaped Wilson lines for hadrons projected to non-zero momentum. By considering non-forward matrix elements of the same operators whose forward matrix elements were studied in Ref. [20] it will thus be possible to include the final state interactions and therefore it will be possible to calculate $\mathcal{L}_{q}$. While there may still be many open issues (e.g. related to renormalization of these matrix elements), even having some insight on the sign of $\mathcal{L}_{q}-L_{q}$ would be very useful at this point.

\section{References}

[1] J. Ashman et al (EMC), Phys. Lett. B 206, 364 (1988); Nucl. Phys. B 328, 1 (1989).

[2] X. Ji, Phys. Rev. Lett. 78, 610 (1997).

[3] Ph. Hägler et al. (LHPC Collaboration), Phys. Rev. D 77, 094502 (2008).

[4] R.L. Jaffe and A. Manohar, Nucl. Phys. B337, 509 (1990).

[5] S.J. Brodsky et al., Nucl. Phys. B 593, 311 (2001).

[6] M. Burkardt and H. BC, Phys. Rev. D79, 071501 (2009).

[7] C. Lorcé and E. Leader, Phys. Rept. 541, 163 (2014).

[8] S. Meissner, A. Metz, and M. Schlegel, J. High Energy Phys. 08 (2009) 056.

[9] A.V. Belitsky, X. Ji, and F. Yuan, Phys. Rev. D 69, 074014 (2004).

[10] X. Ji, X. Xiong, and F. Yuan, Phys. Rev. Lett. 109, 152005 (2012).

[11] A.V. Belitsky, X. Ji, and F. Yuan, Nucl. Phys. B656, 165 (2003); D.Boer, P.J.Mulders, and F.Pijlman, Nucl. Phys. B 667, 201 (2003).

[12] C. Lorcé and B. Pasquini, Phys. Rev. D 84, 014015 (2011); C. Lorce, B. Pasquini, X. Xiong, and F. Yuan, Phys. Rev. D 85, 114006 (2012).

[13] Y. Hatta, Phys. Rev. D 84, 041701 (2011); Phys. Lett. B708, 186 (2012).

[14] S.V. Bashinsky and R.L. Jaffe, Nucl. Phys. B536, 303 (1999).

[15] M. Burkardt, Phys. Rev. D88, 1, 014014 (2013).

[16] J. Qiu and G. Sterman, Phys. Rev. Lett. 67, 2264 (1991).

[17] M. Burkardt, Phys. Rev. D88, 114502 (2013).

[18] D.W. Sivers, Phys. Rev. D 43, 261 (1991).

[19] M. Wakamatsu, Phys. Rev. D 81, 114010 (2010); Eur. Phys. J. A 44, 297 (2010); Phys. Rev. D 85, 114039 (2012).

[20] M. Engelhardt et al., PoS CD12 (2013) 022 